\section{A E E T}

ASOCIACIÓN ESPAÑOLA DE ECOLOGÍA TERRESTRE
Ecosistemas 23(1):1-3 [Enero-Abríl 2014]

Doi.: 10.7818/ECOS.2014.23-1.01

Artículo publicado en Open Access bajo los términos de Creative Commons attribution Non Comercial License 3.0.

MONOGRÁFICO:

Perspectivas en Macroecología: teoría y métodos para

la exploración de patrones y procesos ecogeográficos

\title{
Macroecología: una disciplina de investigación en auge
}

\author{
M.Á. Olalla-Tárraga ${ }^{1, *}$ \\ (1) Área de Biodiversidad y Conservación. Departamento de Biología y Geología, ESCET, Universidad Rey Juan Carlos, c/ Tulipán s/n, 28933 Móstoles, \\ Madrid, España \\ *Autor de correspondencia: M.Olalla-Tárraga [miguel.olalla@urjc.es]
}

> Recibido el 9 de abril de 2014, aceptado el 10 de abril de 2014

Olalla-Tárraga, M.A. 2014. Macroecología: una disciplina de investigación en auge. Ecosistemas 23(1):1-3. Doi.: 10.7818/ECOS.2014.23-1.01

Desde su publicación en 1972, el libro Geographical Ecology de Robert H. MacArthur se ha convertido en una obra clave en el ámbito de la ecología. MacArthur destacó el papel que juega la estructura del ambiente, la morfología y el comportamiento de las especies, las dinámicas poblacionales y la historia a la hora de explicar ciertos patrones ecogeográficos que se manifiestan a grandes escalas espaciales. Quizás la mayor originalidad del trabajo de MacArthur consistió en saber combinar de forma magistral sus conocimientos matemáticos y de historia natural para desarrollar una teoría ecológica explicada a través de simples modelos gráficos y matemáticos. En Geographical Ecology MacArthur aportó una visión extremadamente novedosa al estudio de los patrones de diversidad y distribución de especies y sentó las bases conceptuales y metodológicas de una disciplina que surgiría con fuerza en la década de los noventa: la macroecología (Brown y Maurer 1989).

A lo largo de los últimos veinte años la macroecología, orientada a estudiar patrones y procesos de distribución y abundancia de especies a escalas regionales o incluso globales (Brown 1995), se ha convertido en una disciplina ecológica en auge. A diferencia de aproximaciones metodológicas clásicas en ecología, de carácter eminentemente reduccionista y experimental, la macroecología enfatiza el estudio de patrones estadísticos emergentes a grandes escalas espaciales y temporales. A través del estudio de propiedades emergentes se persigue profundizar en nuestro conocimiento sobre la estructura y el funcionamiento de los sistemas ecológicos, así como tratar de predecir posibles comportamientos futuros. La clave del interés reciente por la macroecología radica probablemente en dos razones principales. En primer lugar, los fuertes vínculos con otras disciplinas científicas como la paleobiología o la macroevolución. Esto ha posibilitado la puesta en marcha de investigaciones interdisciplinares que indudablemente han contribuido a mejorar nuestra comprensión de patrones y procesos naturales (Gaston y Blackburn 2000). En segundo lugar, la escala de análisis de la macroecología coincide con el marco espacial y temporal en el que operan la mayor parte de los componentes del llamado "cambio global" (Kerr et al. 2007). El calentamiento climático, los cambios en los usos del suelo, la fragmentación y pérdida de hábitats, el incremento en la deposición de nitrógeno o la introducción de especies alóctonas tienen importantes efectos a diferentes niveles de la organización biológica, pero operan fundamentalmente a grandes escalas espaciales. Por tanto, se ha hecho evidente la necesidad de planificar estudios macroscópicos que traspasen fronteras políticas y sirvan de complemento a investigaciones de carácter local, que dependen de la manipulación experimental controlada. En este sentido, la macroecología se ha visto favorecida en los últimos años por el desarrollo de herramientas y tecnologías en el ámbito de la teledetección y los Sistemas de Información Geográfica (SIG), así como por la disponibilidad creciente de bases de datos sobre la distribución y biología de especies.

Este Monográfico de la revista Ecosistemas se plantea a raíz de la celebración del Simposio "Avances recientes en Macroecología", que tuvo lugar en el marco del Congreso de la Asociación Española de Ecología Terrestre (AEET) del año 2013. En él se presentaron trabajos tanto a nivel teórico (p. ej. teoría de la conservación filogenética de nichos), como metodológico (p. ej. modelización predictiva de distribución de especies, reglas ecogeográficas, patrones y procesos de diversidad, aplicación de métodos filogenéticos comparativos), o aplicado (evaluación y mitigación de impactos del cambio global) sobre cuestiones macroecológicas. Aunque en el Monográfico no han tenido cabida todos los trabajos presentados a dicho Simposio, los artículos que lo componen son representativos de la variedad de temáticas tratadas.

En el primer artículo, Morales-Castilla y García-Valdés (2014) abordan una cuestión clásica en ecología: ¿cuáles son las causas del gradiente latitudinal de diversidad (GLD)? Desde el siglo XVIII, biogeógrafos y ecólogos han sugerido la existencia de un reducido número de "reglas" ecogeográficas que tratan de "encapsular en forma de patrones la respuesta de faunas y floras de la Tierra a las influencias de factores ambientales" (McDowall 2008). EI GLD, la observación de que la riqueza de especies aumenta hacia los trópicos en la mayoría de los grupos taxonómicos, es considerado por muchos autores como la más antigua y estudiada de estas reglas ecogeográficas (Hawkins 2001). Sin embargo, lejos de lo que cabría esperar, aún hoy estamos lejos de alcanzar un consenso sobre cuál es el mecanismo subyacente a este gradiente. Como sucede en el estudio de cualquier ley natural, las excepciones que prueban la regla suelen ser muy informativas para profundizar sobre posibles causas, motivo por el cual Morales-Castilla y García-Valdés revisan la literatura sobre GLD inversos. Dicha revisión se completa 
con un estudio de caso, el de los mamíferos que participaron en los grandes intercambios bióticos que tuvieron lugar a través del Estrecho de Bering y el Istmo de Panamá a lo largo del Plio-Pleistoceno. Los autores destacan la capacidad explicativa de la teoría de conservación filogenética de nichos climáticos y el tiempo disponible para la especiación, no sólo del patrón regular del GLD, sino de sus excepciones.

A pesar del elevado número de hipótesis ecológicas y evolutivas que han sido propuestas para explicar el GLD [revisadas con detalle por Morales-Castilla y García-Valdés (2014)] las investigaciones orientadas a detectar una posible huella humana sobre los gradientes geográficos de riqueza de especies son muy escasas. En el segundo artículo del Monográfico, Torres-Romero y Olalla-Tárraga (2014) revisan la literatura sobre la relación entre impacto humano y riqueza de especies de vertebrados terrestres a varias escalas espaciales. Del análisis de un total de 29 artículos publicados entre los años 2000 y 2013 concluyen que la mayor parte de los trabajos sobre esta temática se concentran en la región Paleártica, que aves y mamíferos son las dos clases de tetrápodos que han recibido mayor atención y que el signo de la correlación entre impacto humano y riqueza de especies suele cambiar en función del tamaño de grano y la extensión geográfica del estudio. Mientras que a gran escala existe una asociación positiva entre impacto humano (medido generalmente a través de la densidad de población humana) y riqueza de vertebrados terrestres, a escalas locales esta relación se invierte, probablemente debido a procesos de pérdida y fragmentación de hábitat y cambios de uso del suelo.

El sesgo taxonómico que tradicionalmente ha caracterizado los estudios macroecológicos (Beck et al. 2012; Torres-Romero y Olalla-Tárraga 2014) queda patente en el número de artículos del monográfico dedicados a mamíferos. Bini y colaboradores (2014) usan datos de tamaño corporal y área de distribución geográfica de 209 especies del Orden Carnivora a nivel mundial para ilustrar el funcionamiento de un nuevo método filogenético comparativo llamado Curva de Señal-Representación Filogenética (PSR). El método PSR es una expansión de la regresión basada en autovectores filogenéticos (PVR; Diniz-Filho et al. 1998) que ha sido ampliamente utilizada para analizar datos ecológicos en un marco evolutivo. Las curvas PSR pueden ser usadas en un contexto exploratorio de autocorrelación filogenética y son muy útiles para visualizar gráficamente patrones filogenéticos en la variación de rasgos biológicos bajo diferentes modelos evolutivos. Avanzar en nuestra comprensión sobre la distribución geográfica de la biodiversidad requiere integrar abordajes macroecológicos y macroevolutivos construyendo puentes conceptuales y desarrollando este tipo de técnicas analíticas.

En esta misma línea de combinar métodos filogenéticos comparativos con datos macroecológicos, Martínez y colaboradores (2014) recopilan datos morfológicos de especies en una familia de mamíferos carnívoros (Felidae) para estudiar la regla de Rensch. Esta regla ecogeográfica propone que existe una relación alométrica entre el dimorfismo sexual de tamaño y el tamaño corporal medio. En taxones en los que los machos son mayores que las hembras se esperan aumentos del dimorfismo sexual de tamaños con incrementos de tamaño medio de las especies, y a la inversa si las hembras son mayores. Aunque encuentran que los félidos muestran elevados niveles de dimorfismo sexual en la masa corporal, no se verifica la validez de la regla de Rensch en esta familia. Del mismo modo, ninguno de los predictores climáticos, ecológicos y de rasgos de historias de vida examinados mostró una asociación con el dimorfismo sexual de tamaño. Se trata de un estudio interespecífico y los autores apuntan a la necesidad de trabajar a otro nivel de la organización biológica, examinando la variación intraespecífica de tamaños en especies con amplios rangos de distribución geográfica, para poder profundizar en los posibles mecanismos moduladores de los patrones de variación morfológica observados.

El clima, y en especial la temperatura, juegan un papel fundamental en la determinación de los límites del rango de distribución geográfica de las especies. En vertebrados ectotermos como los reptiles esta señal climática suele ser especialmente pronunciada. Cruz y colaboradores (2014) utilizan datos de la ecología térmica y la distribución geográfica de 47 especies de lagartos del género Liolaemus de Sudamérica para evaluar tres hipótesis que tratan de explicar la respuesta de las especies a gradientes geográficos de temperatura. No encuentran apoyo para la hipótesis de variabilidad climática, según la cual organismos a latitudes más altas mostrarían mayores niveles de tolerancia térmica como respuesta a la mayor estacionalidad de las temperaturas ambientales. Sin embargo, sí se corrobora la relevancia de la elevación como indicador de viviparismo en reptiles. De acuerdo con estudios recientes para otros reptiles (véase por ejemplo Meiri et al. 2013), las especies vivíparas de Liolaemus soportan temperaturas ambientales promedio en torno a $5^{\circ}$ inferiores a las experimentadas por especies ovíparas. Asimismo, las hembras de las especies vivíparas de Liolaemus estudiadas mostraron una mayor capacidad termoreguladora que las ovíparas. La temperatura ambiental es, por tanto, un parámetro determinante para la distribución geográfica de estos organismos.

Los modelos predictivos de distribución de especies (MDE) se fundamentan precisamente en la relevancia del clima como limitante del rango de distribución geográfica. Los MDE son herramientas ampliamente usadas en macroecología que permiten estimar la distribución real y potencial combinando datos sobre el nicho ecológico de las especies con información geográfica. Los dos últimos artículos del Monográfico están referidos a cuestiones metodológicas sobre la implementación de estos modelos. Varela y colaboradores (2014) exploran mediante la creación de especies virtuales cómo los MDE pueden verse afectados por datos erróneos y sesgos de muestreo que aparecen con relativa frecuencia en las grandes bases de datos que contienen registros de presencia de las especies (p.ej. GBIF). Se comparan los resultados de dos algoritmos de modelización ampliamente usados: Bioclim y Maxent. Mientras que el primero parece producir estimas más fiables de área potencial cuando los datos de calibración contienen sesgos pero no errores, Maxent parece ser menos sensible a la presencia de errores. Este trabajo deja abierta la puerta a realizar una comparación más completa con otro tipo de técnicas (ej. ENFA, GLM, GAMs, etc.). En ese sentido, Ropero y colaboradores (2014) resaltan en el último artículo del Monográfico la potencialidad de las redes bayesianas como herramientas estadísticas en MDE. Su uso apenas está extendido en este ámbito, a pesar de las ventajas y posibilidades que ofrecen en el manejo de datos discretos, continuos o híbridos. Se describe, en función del objetivo del modelo (caracterización, clasificación y regresión), la aplicación de las redes bayesianas al ámbito de los MDE, ilustrándola con ejemplos de los diferentes métodos de aprendizaje y construcción del modelo. Los autores resaltan que quizás la mayor ventaja de esta aproximación con respecto a otras técnicas de modelización estribe en la facilidad de trabajar con escenarios futuros. En un contexto de cambio global, estas soluciones procedimentales son especialmente bienvenidas.

\section{Referencias}

Beck, J., Ballesteros-Mejia, L., Buchmann, C.M., Dengler, J., Fritz, S.A., Gruber, B. et al. 2012. What's on the horizon for macroecology? Ecography 35: 673-683.

Bini, L.M., Villalobos, F., Diniz-Filho, J.A.F. 2014. Explorando patrones en rasgos macroecológicos utilizando regresión secuencial de autovectores filogenéticos. Ecosistemas 23(1):000-000. Doi.: 10.7818/ECOS.2014.23-1.04

Brown, J.H. 1995. Macroecology. Chicago, University of Chicago. Chicago, Estados Unidos. 269p.

Brown, J.H, Maurer, B.A. 1989. Macroecology: The division of food and space among species on continents. Science 243: 1145-1150.

Cruz, F.B., Moreno Azócar, D.L., Bonino, M.F., Schulte II, J.A., Abdala, C.S., Perotti, M.G. 2014. Clima, distribución geográfica y viviparismo en especies de Liolaemus (Reptilia; Squamata): cuando las hipótesis se ponen a prueba. Ecosistemas 23(1):00-00. Doi.: 10.7818/ECOS.2014.23-1.06 
Diniz-Filho, J.A.F., Sant'Ana, C.E.R., Bini, L.M. 1998. An eigenvector method for estimating phylogenetic inertia. Evolution 52: 1247-1262.

Kerr, J.T., Kharouba, H.M., Currie, D.J. 2007. The Macroecological Contribution to Global Change Solutions. Science 316: 1581-1584.

Gaston, K.J., Blackburn, T. 2000. Pattern and Process in Macroecology. Blackwell Science, Cambridge., Reino Unido.

Hawkins, B.A. 2001. Ecology's oldest pattern? Endeavour 25: 133-134.

Martínez, P.A., Ferreira Amado, T., Bidau, C.J. 2014. Una aproximación filogenética al estudio del dimorfismo sexual de tamaño en Felidae y la evaluación de la regla de Rensch. Ecosistemas 23(1):000-000. Doi.: 10.7818/ECOS.2014.23-1.05

McDowall, R.M. 2008. Jordan's and other ecogeographical rules, and the vertebral number in fishes. Journal of Biogeography 35: 501-508.

Meiri, S., Bauer, A.M., Chirio, L., Colli, G.R., Das, I., Doan, T.M., Feldman, A., Castro Herrera, F., Novosolov, M., Pafilis, P., Pincheira-Donoso, D., Powney, G., Torres-Carvajal, O., Uetz, P., Van Damme, R. 2013. Are lizards feeling the heat? A tale of ecology and evolution under two temperatures. Global Ecology and Biogeography 22: 834-845.

Morales-Castilla, I., García-Valdés, R. 2014. Gradientes latitudinales de diversidad inversos, ¿excepciones que prueban la regla. Ecosistemas 23(1):000-000. Doi.: 10.7818/ECOS.2014.23-1.02.

Ropero, R.F., Aguilera, P.A., Fernández, A., Rumí, R. 2014. Redes bayesianas: una herramienta probabilística en los modelos de distribución de especies. Ecosistemas 23(1):000-000. Doi.: 10.7818/ECOS.2014.23-1.00.

Torres-Romero, E.J., Olalla-Tárraga, M.A. 2014. Impacto humano y riqueza de especies de vertebrados terrestres: una revisión a diferentes escalas macroecológicas. Ecosistemas 23(1):000-000. Doi.: 10.7818/ECOS.2014.23-1.03.

Varela, S., Mateo, R.G., García-Valdés, R., Fernández-González, F. 2014. Macroecología y ecoinformática: sesgos, errores y predicciones en el modelado de distribuciones. Ecosistemas 23(1): 000-000. Doi.: 10.7818/ECOS.2014.23-1-07. 